\title{
Test-bed Development \& Measurement Plan for Evaluating Transmit Diversity in DVB Networks
}

\author{
Shirazi $\mathrm{H}^{1}$, Di Bari $\mathrm{R}^{1}$, Cosmas $\mathrm{J}^{1}$, Nilavalan $\mathrm{R}^{1}$, Zhang $\mathrm{Y}^{1}$, Loo $\mathrm{J}^{1}$, Bard $\mathrm{M}^{2}$ \\ 1- Brunel University, Uxbridge, Middlesex, UK; 2- Broadreach Systems, Devon, UK
}

\begin{abstract}
This paper presents a test-bed development and measurement plan for evaluating transmit diversity in the DVB Network. Transmit diversity reduces the complexity and improves the power consumption of the personal receiving devices by improving the transmission of signals in NLOS cluttered environments. Also, it is more practical than receive diversity due to the difficulty of locating two receive antennas far enough apart in a small mobile device. Test service scenarios were developed to illustrate the benefits of such technologies so that effectiveness can be researched in a variety of service and terrain scenarios using purpose built test systems. The laboratory tests were designed to validate the theoretical measurements from the theoretical analysis and these results will be verified by a field measurement campaign in short and long time spans.
\end{abstract}

Index terms - CDD, DD, SISO, SIMO, MISO, MIMO, Field trials.

\section{INTRODUCTION}

The multiple transmit and receive antennas (Multiple InputMultiple Output - MIMO) is a well-documented technique where each pair of transmit and receive antennas provides a different signal path from the transmitter to the receiver. Receiving the same information from multiple paths would reduce the likelihood of deep fading by exploiting the statistical nature of fading. In fact, the measurement results of simulations confirm that the diversity techniques will improve reception in areas of poor coverage [1] [3]. These techniques can be applied to DVB-T/H and DAB systems that use Orthogonal Frequency Division Multiplexing (OFDM). For standardized systems, only additional spatial diversity techniques can be implemented, since these modifications keep the systems standards compatible.

The implementation of the receive diversity in the existing hand-held devices is sometimes impractical due to the problem of situating the antennas sufficiently far apart for UHF band reception. However, the transmit diversity does not impose these issues whilst still being compatible with the existing hand-held devices. It has been shown that transmit diversity techniques like Cyclic Delay Diversity (CDD) and Delay Diversity (DD) can improve the performance of the existing broadcasting systems although their effect is yet to be measured in the field. For this purpose, the Physical Layer DVB Transmission Optimisation (PLUTO) European project is developing a demonstrator for taking field measurements. Even though the project is focused on the transmit diversity, some measurements for receiving techniques are planned to compromise both configurations.

The measurement campaign will be focused mostly in places where the OFDM system presents poor performances (i.e. indoors and sparsely populated or obscured locations). It benefits directly from the special Diversity equipments employed in the test-bed and make directly measurement of parameters like the Received Power, SNR and all the DVB$\mathrm{T} / \mathrm{H}$ Bit Error Rate set. The results of the experiments will validate the improved system performance predicted through simulation results obtained in [1] [3] [7].

The test-bed plan will be arranged into two parts: the short term and the long-term measurement campaigns.

In the short-term measurement campaign, it is planned to build a novel system to verify the performance of diversity in the DVB-T/H system via laboratory and the field tests. In the preliminary laboratory tests, the accuracy of the new equipments, which are developed specially in this project for providing diversity, are tested and calibrated for a first benchmark of the theoretical results. Then, the field tests will provide the definitive figures of diversity gain in the real environments.

In the long-term measurement campaign, the objective is to analyse the effect of diversity techniques on the DVB system over a long period of time through a monitoring centre consisting of more than one receiver located in different places. The monitoring centre provides the broadcast operator with the data indicating whether the diversity techniques alleviate the combined effects of foliage, weather conditions and seasonal changing. This is a centralised system to manage and monitor remotely all the receiving devices and store the measured data over a long time.

The paper is structured as follows. The second section describes the theoretical activities underlining the gain of the diversity in the DVB system. In the third section the measurement framework is given in which the type of environment, antenna positioning and measurement parameters are defined. The forth section is focused on shorttime measurements made in the laboratory and field tests. It presents the system diagram and explanation of the apparatus. The fifth section describes the long-term measurements. It presents the system and network measurement diagram. The Network Monitoring sub-system is introduced in this section and the post-processing tasks are explained. The sixth section presents the paper's conclusions.

\section{THEORETICAL GUIDELINES}

In this section, some theoretical results are presented as general guidelines for transmit diversity system design.

To achieve transmit diversity gain, the signals from different antenna elements should be sufficiently uncorrelated (at least less than 0.7 correlation). Moreover, the transmitted signals should ideally have equal average power (less than $3-\mathrm{dB}$ 
difference). Many measurement and experimental results show that when two antennas are used to provide diversity gain, a minimum separation of ten wavelengths is required at the transmitter [6].

An example of the above-mentioned results is illustrated in Figure 1, where the cross correlation function versus $2 \times 1$ base station antennas spacing and the Ricean $\mathrm{K}$ factor is plotted

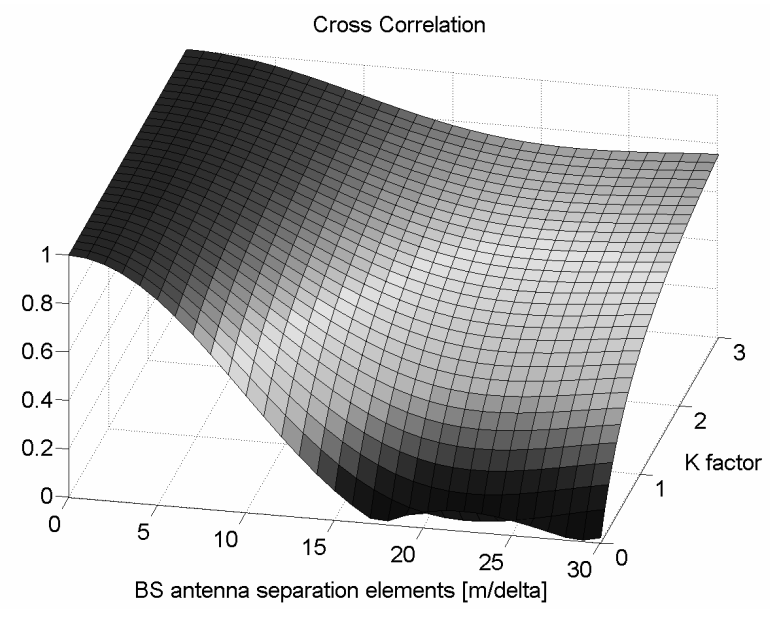

Figure 1: Simulation of Cross correlation Vs. the BS antenna spacing and the Ricean $\mathrm{K}$ factor

When Ricean K factor is about zero (NLOS), the minimum cross correlation of 0.7 is achieved by separating the transmit antennas by almost 7 wavelengths. The cross correlation reduces to almost zero when the antenna elements are separated by at least 16 wavelengths. This result matches well with the previous predictions of the required separation for base station antennas. To get similar channel decorrelation with increasing values of the $\mathrm{K}$ factor (LOS), higher antenna separations are required. For instance, for $\mathrm{K}=3$, the cross correlation is less than 0.7 with about 20 wavelengths antenna separation.

To maintain a same level of channel decorrelation, higher antenna separation is required as $\mathrm{K}$-factor increases. The transmit diversity techniques are effective in the NLOS situations where the K-factor is small valued [5].

Figure 2 and Figure 3 show the transmit diversity gain in DD obtained from simulation. The simulation was carried out on QPSK modulation over DVB-H 4k mode. Figure 2 presents the BER versus SNR over diverse levels of channel cross correlation adopting the COST 207 Rural Area channel [7]. Figure 3 shows the impact of the Ricean $\mathrm{K}$ factor on the diversity performances while adopting the COST 207 Typical Urban channel.

These results simply verify the loss of the diversity gain while Ricean $\mathrm{K}$ factor increases [9].

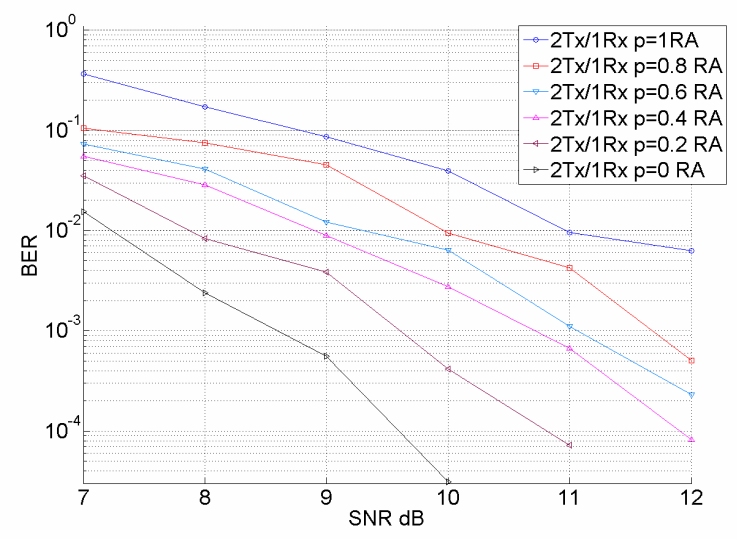

Figure 2: BER vs. SNR for DVB-H 4k mode QPSK for Rural Area correlated channels.

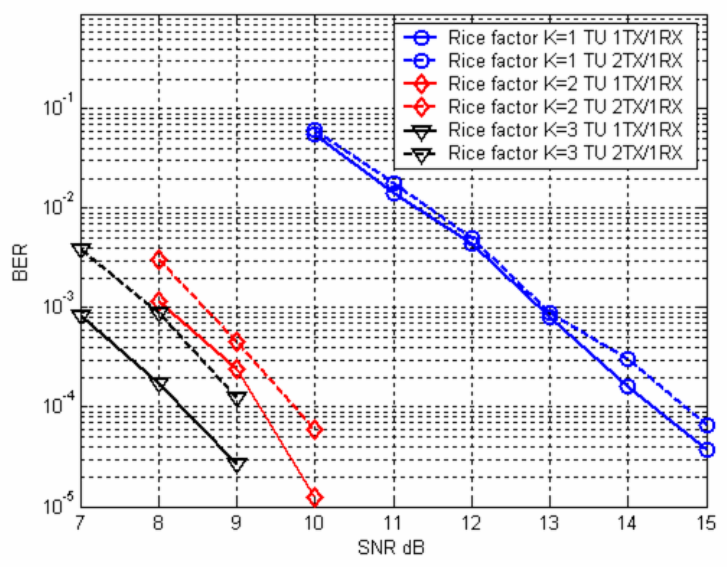

Figure 3: BER vs. SNR for DVB-H 4k mode QPSK for Typical Urban for different values of Ricean $\mathrm{K}$ factor.

\section{MEASUREMENT FRAMEWORK}

In the PLUTO project, one of the test-field measurements takes place in the sites located around the Brunel University (Uxbridge, UK) setting the DVB-T UK mode (2k, 16 QAM, $\mathrm{CR}=3 / 4$, GI $=1 / 32$ ). The signal is transmitted from a university building in the UHF frequency band $(730 \mathrm{MHz})$ with $20 \mathrm{~dB}$ power due east. The antenna system in the base station includes fives antennas (ADC broadband panel) arranged as shown in Figure 4 (next page). The maximum space to mount the antennas horizontally is $24 \lambda$, and vertically is $12 \lambda$. Also, the maximum of $27 \lambda$ separation is achieved by combination of horizontal and vertical spacing. Therefore, the antenna elements separation is enough to achieve different level of cross correlation by transmitting through two antennas at a time.

The signals are space-time coded based on CDD or DD techniques before being transmitted. According to the previous section, this set-up will guarantee enough decorrelation of the two-diversity channel for promising diversity gains. 


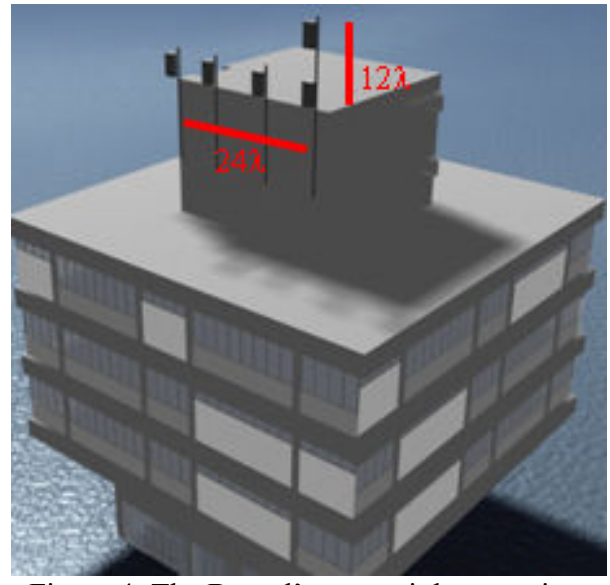

Figure 4: The Brunel's transmit base station.

The environment around the Brunel transmit site is classified as semi-rural due to the student residential halls, campus buildings, houses and tree coverage. The measurements are mainly made in the indoor (offices), rooftop situations.

The simulation results reveal that the transmit diversity techniques improve the quality of signal in NLOS situation and deteriorate in LOS [5]. Therefore, it is desirable, especially for an operator, to find out a compromise of applying the transmit diversity techniques to satisfy both LOS and NLOS situations.

In order to verify the diversity gain, the following parameters need to be measured:

1. MER, BER before Viterbi, Viterbi BER and DVB-H error set (RSBER, RSFER and RSSER)

2. Signal to Noise Ratio (SNR)

3. Cross Correlation between two transmit antennas

4. Receiver location

5. Receiver time

6. Radio Frequency (RF) Power level

7. Channel Impulse Response (CIR)

After defining the environment type, antenna system arrangement and the requisite parameters, the set-up of apparatus and post-processing steps in the laboratory and field tests are given in the following sections, where the short-term and long-term measurements are expanded.

\section{THE SHORT-TERM MEASUREMENT DESIGN}

In the short-term measurements, it is planned to set up a system to validate the performances of diversity in the DVB$\mathrm{T} / \mathrm{H}$ via laboratory and the field tests. In the preliminary laboratory tests, the new equipments, which were specially developed to monitor the signal and provide diversity, are tested and calibrated to be used for a first benchmark of the theoretical results. Then, the field tests will provide the definitive figures of diversity gain in the real environments.

\section{A. Lab test design}

The laboratory test system is designed to test the accuracy of the equipments in an emulated real-world deployment conditions. The equipments are used in the subsequent field measurements campaigns. Figure 5 shows the block diagram of the system architecture in the laboratory environment.

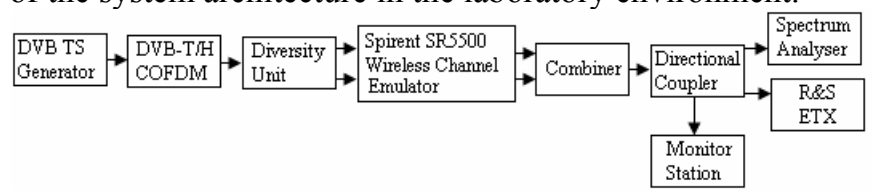

Figure 5: The system layout of the laboratory environment.

In laboratory environment, audiovisual signals are encoded into the MPEG-2 stream s. The multiplexer multiplexes the programmes and sends the transport streams to the DVB-T modulator. The RF signals are then fed either directly into a channel emulator (e.g. Spirent SR5500), where the characteristics of various channels like Rice, Gaussian and Rayleigh are emulated, or through a MIMO Diversity device to investigate the performance of the different diversity techniques on the quality of signal. Moreover, it is used for comparing the laboratory results with predicted simulation results and to ascertain any limitation of the implementation of the real system within the controlled environment. Finally the directional coupler leads the faded signals into the signal analysers representing the professional, industrial and experimental technologies.

The Diversity Unit encodes the modulated signal using the Delay Diversity techniques and conditions it using the Delay Diversity Techniques [5]. The modulated signals are measured in the HP 8560E Spectrum Analyzer and two receivers - the Rohde and Schwarz ETX and Monitor Station During the laboratory observations, the first analysis will be probing and calibrating the Diversity Unit according to the industrial standards. For this end, the R\&S ETX and Spectrum Analyser are used as a reference. These instruments, respectively, provide the measurements of the channel estimation and the received signal affected by echoes. In this way, the accuracy of the Monitor Station will be checked. Thanks to the adoption of the channel emulator, it is possible to emulate all the previously emulated experiments with different channel models (Rice, Rayleigh and etc.). Also, the working receiver's limits of operation can be obtained through studying the SNR and Doppler frequency, and the impact of the channel estimation error on the system performance can be identified. Finally, it is planned to perform series of tests to study the theoretical benefits of the transmit diversity regarding the channel cross correlation and system performance.

After having tested the performance of the newly developed equipment and verified simulation results in a small-scale laboratory environment, the behaviour of the diversity techniques on the DVB system in the real world will be studied. The next section describes the measurement plan, apparatus description and post-processing functions for the short-time-span measurement campaign.

\section{B. Field- test design}

In this section the design of short-term measurement campaign is described. The main objective of this part is to measure the gain of the diversity techniques over various 
system configurations. Figure 6 shows the block diagram of the system architecture.

At the transmit side, the diversity unit feeds MPEG-2 signals into two power amplifiers. Then, the signals are led to the equally powered transmitter antennas. The maximum of two antennas (out of five mounted antennas) are active at any time. At the receiver side, the DVB receiver, GPS, a computer PC and power supply are connected to each other. The Rohde \& Schwartz ETX receiver, Monitor Station and DIBCOM receiver diversity unit are the receiver alternatives available in this measurement campaign. The Monitor Station and DIBCOM receivers incorporate the Maximum Ratio Combining (MRC) technique make it possible to run and test the performance of the receive diversity technique. The receiver apparatus is either set in a fixed position or carried around on a buggy or car to study performance in stationary slow fading and moving fast fading environments.

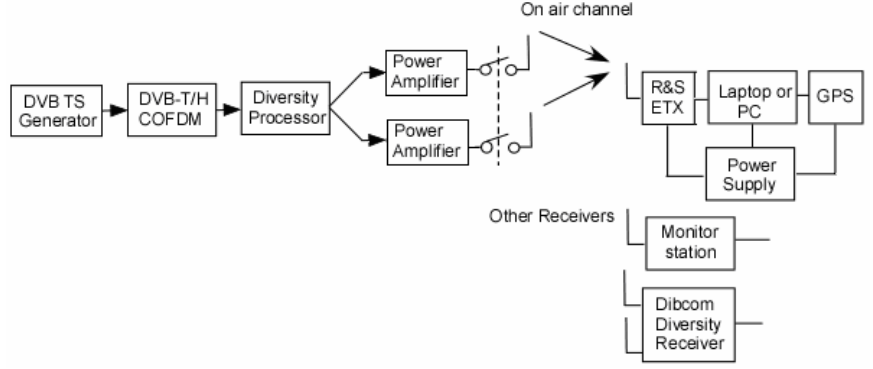

Figure 6: The layout of the short-term measurement design.

The design of the system is required to prove and measure the benefits of all the diversity configurations (MISO, SIMO, MIMO) in typical reception environments (such as indoor, rooftop, driving and etc.)

\section{Measurements and post-processing}

As mentioned before, the measured data include RF received power, SNR, channel impulses responses and all the DVB$\mathrm{T} / \mathrm{H}$ Bit Error Rates at different transmit and receive antennas set-up. They are used to directly study the benefits of diversity in the real environments. In fact, it is desirable to establish the relationship between the system performance and diversity techniques for each environment.

Each environment can be characterized by the corresponding measured channel impulse response (CIR). A large number of CIR data needs to be stored and processed for each environment. A first analysis of the stationary channel allows us to choose the correct averaging procedure and to model a representative power delay profile. The aim is to determine the appropriate model from the most used statistical channel models (i.e. COST 207, COST 273 and Saleh-Valenzuela), that best fits the channel models resulting from measurements. Subsequently, a key function will provide the information regarding the cross-correlation between diversity channels. The derived data will be completed with function outputs that statistically characterize the other fundamental channel properties including the Number of Taps, maximum Channel Delay, the distance-power law attenuation coefficient, the Doppler Spectrum Form, time/frequency distribution of Fading and the Ricean $\mathrm{K}$ factor. This information is utilised to quantify the impact of the novel techniques to reduce the diversity loss in the LOS situation.

At this point, each power delay profile is associated with the measured diversity gains to provide typical information of the achievable diversity gain for each environment.

In the next section a system is given to evaluate the gain of diversity techniques on the DVB system over a long period.

\section{THE LONG-TERM MEASUREMENT DESIGN}

In the long-term measurements, the objective is to identify the effect of diversity techniques on the performance of the DVB system over a long-time-spin. To fulfil the measurements, a monitoring system is introduced that automatically controls and collects the measured data from one or many receiver(s) located in different areas. Also, it enables us to measure the gain of the diversity techniques over the combined effects of foliage, weather conditions and seasonal changing.

\section{A. System test design}

The long-term measurement campaign is designed to monitor and investigate the effects of diversity techniques on the quality of the signal in the DVB network over long time periods. This scheme is an extension of the short-term measurement system described in the previous section. Taking into consideration the scope of the measurement campaign, a system is required to automatically conduct the tests over a long period of time. Figure 7 (next page) shows the design of the long-term data collection system.

The system consists of the transmit equipment used in the short-term measurement and a number of receivers located within the range of radio coverage in the sites characterised previously by the short-term trials. The receiver is connected to the outdoor weather station (meter), which provides the temperature, pressure, humidity and wind speed information. Also, it is connected to the IP network to send the measurement report periodically to the Monitor Centre, which is a system that has been designed to manage one or more Monitor Station(s) in the PLUTO project. It incorporates the Java applications and SQL database. The applications execute the control, monitor and post-processing tasks; such as measurement report collection and experimental parameters configuration, plus data retrieval from and store to the database. The operator initiates the experiments through this system by sending the measurement configuration to each registered Monitor Station. Then, it periodically collects the reports related to each experiment. Knowing the source of the reports, the Monitor Centre stores and classifies the information for post-processing. Also, it utilises the Simple Network Management Protocol (SNMP) and an alarm system to inform the monitor station of any significant change in the measurement results and an outcome of some exceptional seasonal or weather changes in the environment.

The Monitor Centre provides the web services that enable the operator to remotely follow up the measurement progress and performing post-processing functions. 


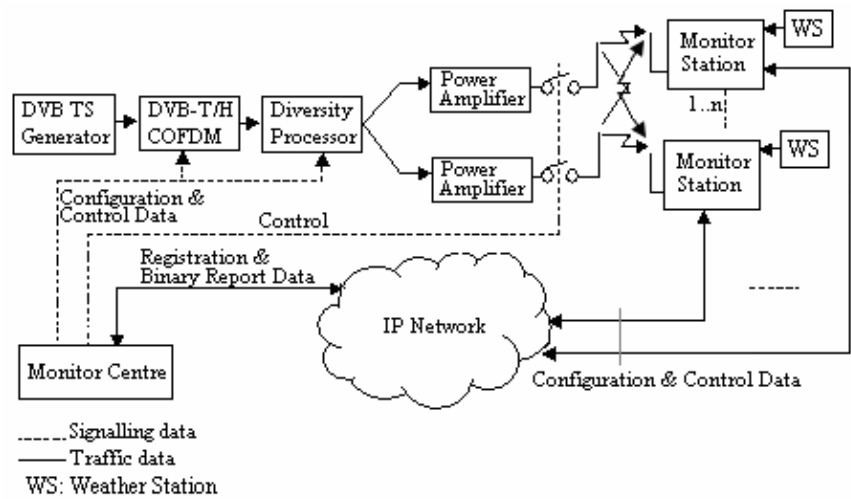

Figure 7: The layout of the Long-term Measurement System.

The next section describes the measurements and postprocessing objectives.

\section{B. Measurements and Post-processing}

The long-term measurements is planned to validate the gain of the diversity techniques in DVB system under the effects of various seasonal and weather conditions. Therefore, the information stating the climate conditions needs to be measured and stored beside the other measurement parameters foresaid. These data include general weather condition (wind speed, rain, etc), classification of the test site according to the tree coverage, the level of Fresnel zone shadowing, and foliage percentage. In the post-processing, the measured data is segmented and averaged every, for instance, 30 minutes.

The measurements are carried out mainly in the indoor and rooftop situations. As mentioned earlier, the rooftop scenario is highly considered as LOS situation, and the Ricean $\mathrm{K}$ factor is a fundamental parameter at the diversity performances. Thus, it is also desirable to find out the correlation of $\mathrm{K}$ factor and the climate changes. In fact, it has been established that $\mathrm{K}$ factor highly depends on the median excess path loss, average wind speed and season moving from summer (more leaves) to winter (less leaves) [2]. Another parameter that affects the reception is the atmosphere precipitations. Raindrops cause greater attenuation by scattering than by absorption at frequencies above $100 \mathrm{MHz}$ [2]. Hence, the first approach in the measurement is to correlate the path loss with the Ricean $\mathrm{K}$ factor, then compare the benefit of diversity versus such deteriorations in the quality of signal.

\section{CONCLUSION}

In this paper, the measurement guidelines, test-bed developments and measurement plans for evaluating transmit diversity in the DVB Network has been presented. The design of the demonstrator system has been discussed. The parameters that need to be measured and post processed have been identified.

The demonstrator has been designed to validate the simulation results regarding the gain of at least $5 \mathrm{~dB}$ realized via using the space-time coding at the transmitter. The design of the presented test-bed is able to test transmit diversity techniques that can potentially accommodated by existing
DVB broadcasting system without changing the standards or the receivers with an arbitrary number of separated transmit antennas.

The resulting digital broadcast network would have fewer transmitter sites and thus be more cost-effective and have less environmental impact.

\section{REFERENCE}

[1] Yue Zhang; Cosmas, J.; Bard, M.; Yong-Hua Song : "Diversity gain for DVB-H by using transmitter/receiver cyclic delay diversity", IEEE Transactions on Broadcasting, v 52, n 4, Dec. 2006, p 464-74

[2] Crosby, D. ; Abhayawardhana, V.S.; Wassell, I.J.; Brown, M.G.; Sellars, M.P.: " Time variability of the foliated fixed wireless access channel at $3.5 \mathrm{GHz}$ ", 2005 IEEE 61st Vehicular Technology Conference. VTC2005- Spring (IEEE Cat. No. 05CH37631), 2005, pt. 1, p 106-10 Vol. 1

[3] A. Dammann and S. Kaiser, "Standard conformable antenna diversity techniques for OFDM systems and its application to the DVB-T system," in IEEE Globecom, pp. 3100-3105, November 2001.

[4] A. Abdi, M. Kaveh, "A space-time correlation model for multielement antenna systems in mobile fading channels", IEEE Journal on Selected Areas in Communications, v 20, n 3, April 2002, p 550-60

[5] Armin Dammann, Ronald Raulefs. Report on Measurement Metrics and Theoretical Diversity Modelling. PLUTO Deliverable 4.1 August 2006.

[6] S. M. Alamouti, "A Simple transmit diversity technique for wireless communications." IEEE J. Select. Areas Communications., vol. 16,pp.1451-1458, Oct. 1998

[7] Yue Zhang, John Cosmas, Maurice Bard and YongHua Song "Future Transmitter/Receiver Schemes in Broadcast Wireless Networks" IEEE Communications Magazine, October 2006

[8] Y. Zhang, J. Cosmas, M. Bard, Y.H. Song, K.K. Loo and R.D. Bari: "Analysis of Cyclic Delay Diversity on DVB-H Systems over Spatially Correlated Channel" Accepted in IEEE Broadcast Transactions.

[9] Y. Zhang, J. Cosmas, K. K Loo and M. Bard, "Performance Analysis of Combined CDD and Discontinuous Doppler Diversity in Rician and Rayleigh Channels". Submitted to 16th IST Mobile and Wireless Communications Summit 2007. 